\title{
Propuesta de un Modelo de Evaluación para Fortalecer la Profesión Docente en los Niveles Obligatorios. El Caso de Asturias (España)
}

\author{
A Proposal of a Model of Evaluation as an Element to Stregthen \\ the Teaching Profession at the Compulsory Levels. \\ The Case of Asturias (Spain)
}

\author{
Blanca Arteaga-Martínez* y Josu Ahedo-Ruiz \\ Universidad Internacional de La Rioja (UNIR)
}

\begin{abstract}
La evaluación docente debe contar con un enfoque adecuado para mejorar la calidad académica. En este artículo se presenta una propuesta de cuáles son los elementos a evaluar, desde un planteamiento que acentúa la formación del profesorado como factor clave para motivar al estudiante en su aprendizaje.

Desde los objetivos que se plantean en el proceso de enseñanza se justifica la conjunción entre la formación y la evaluación del docente. Se analiza el modelo de Asturias, el primero que se aprueba en España, basado en la evaluación por incentivos, detallando las competencias evaluadas y los datos obtenidos en los últimos años. A modo de conclusión, se subraya la necesidad de la aprobación de un estatuto docente consensuado con los docentes como solución frente a la burocratización de la profesión, rechazada por los docentes. El diseño de este estatuto debe contar con el profesorado y con las familias de los estudiantes.
\end{abstract}

Palabras clave: Desempeño docente, Evaluación docente, Asturias, Formación profesorado, Estatuto docente.

The improvement of academic quality requires a right approach to the assessment of teaching practices. This article makes a proposal of the evaluative features to be considered, from a perspective that stresses teacher training as a key factor to motivate students.

Since the aims that it be planted in the process of teaching, it explains the conjunction between the formation and teacher evaluation. It analyzes the model of Asturias, the first that is approved in Spain, based in the evaluation for incentives, detailing the evaluated competences and the data obtained in the last years. By way of conclusion it underlines the necessity of approving one teaching statute agreed wih the teachers as a solution front of bureaucratization of the profession, rejected by teachers. This statute must be designed with the teachers and with the students families.

Keywords: Teaching performance, Teacher evaluation, Asturias, Teacher training, Teaching statute.

*Contacto: blanca.arteaga@unir.net

issn: $1989-0397$

www.rinace.net/riee/

https://revistas.uam.es/riee
Recibido: $\quad 8$ de abril de 2016

$1^{\text {a }}$ Evaluación: 17 de junio de 2016

Aceptado: $\quad 15$ de julio de 2016 


\section{Introducción}

El presente artículo tiene como propósito justificar la posible necesidad de definir un marco normativo regulador en España, habida cuenta de cómo es la evaluación del docente en los niveles educativos previos a la universidad. En primer lugar, analizaremos el papel de la evaluación del docente en un contexto donde los resultados no dependen únicamente del ejercicio de su profesión, sino de otros factores relacionados con las características del centro educativo —organización, clima, recursos, etc.- y de la actitud y aptitud del estudiante, tanto de forma individual como en grupo.

Este análisis permite presentar un modelo para la evaluación del docente, subrayando que la evaluación ha de plantearse como mejora de la calidad educativa, integrada en el plan de evaluación del centro y que proporcione unos "profesores competentes, motivados, considerados socialmente y bien dispuestos a la innovación" (Gimeno, 2010, p. 244).

La evaluación del docente se ha de centrar en la práctica de este, pero también en los resultados de rendimiento de los estudiantes, evitando conclusiones generalizadoras. Actualmente, las prácticas de evaluación combinan distintas fuentes de obtención de datos. Se realiza una exposición del caso de Asturias, pionera en la regulación de la evaluación de los docentes en los niveles educativos obligatorios previos a la universidad. Esta regulación hace realidad un sistema de incentivos que, como proceso aislado en el territorio español, pone en evidencia la necesidad de un estatuto docente que facilite la promoción y formación de los docentes.

\section{La evaluación del desempeño docente}

Las "cambiantes expectativas de las economías del conocimiento" (OCDE, 2009, p. 12) en los diferentes países sitúan como una necesidad avalar el planeamiento de políticas relacionadas con la calidad de sus sistemas educativos, para hacer frente a las exigencias de una sociedad futura. Esto implica la pertinencia de que los diseños formativos incluyan la mejora continua de sus procesos, sistematizando elementos evaluadores de aquellas situaciones y agentes implicados en la formación de sus ciudadanos. Esta sistematización debería tener en cuenta las dos funciones fundamentales en la evaluación señaladas por Scriven (1967), la formativa y la sumativa.

El término evaluación carece de ambigüedades y señala el valor de algo mediante la emisión de un juicio (Gimeno y Pérez, 1989). La evaluación debe tener claras diferentes premisas en su diseño que permitan garantizar que se tienen en cuenta todas las dimensiones susceptibles de ser medidas. Por ejemplo, no es conveniente medir el aprendizaje de los estudiantes observando únicamente el resultado de su acción individual, sino "la articulación de las actividades del profesor y de los alumnos en torno a un contenido o una tarea de aprendizaje" (Coll, Colomina, Onrubia y Rochera, 1992, p. 191).

La evaluación en el entorno educativo es compleja dada la amplitud de escenarios, agentes, situaciones, etc., cuya diversidad de circunstancias dificulta encontrar una fórmula común que abarque a todos. En este sentido, Anderson (2005) señala tres tipos de sistemas para llevar a cabo la rendición de cuentas (accountability). El primero basado 
en el cumplimiento de regulaciones - podríamos hablar en este punto de la burocracia educativa, ineludible desde la necesidad de equidad dentro del sistema-; el segundo, en la adhesión a los estándares profesionales — se asocia tanto a la mejora formativa de los docentes como al ajuste educativo que se brinda al estudiante-; y el tercero, en los resultados —en términos de rendimiento, asociados a logro académico y con potencial para establecer rankings entre escuelas y sistemas-.

Esta complejidad se acrecienta porque la docencia es una actividad humana y como tal tiene un carácter complejo y polémico (Rueda, 2009). En este sentido, De la Orden (2012) señala que conviene clarificar el sentido, significado, alcance y función de la evaluación con el fin de promover la calidad de la educación. Según Stiggins y Duke (1988), la evaluación es esencial por dos propósitos básicos: la responsabilidad y el desarrollo profesional, aspectos integrados dentro de la cultura profesional del docente que deben formar parte de un proceso global (Imbernón, 1994).

El objetivo de la evaluación docente debe centrarse en el perfeccionamiento educativo, basado en la mejora del proceso y los resultados, fundamentado en el análisis y evaluación de políticas e innovaciones educativas (De la Vega, 2015). Asimismo, parece existir consenso en que la recogida de resultados objeto de análisis se ha de realizar en el aula, escenario en el que se establece la relación entre estudiante, docente y contenido, y al que Houssaye (1993) denominó triángulo pedagógico y Elmore (2010), años más tarde, núcleo pedagógico.

Sin embargo, resaltamos la insuficiencia de este análisis porque en el escenario educativo español uno de los principales retos es enlazar la práctica y la teoría dentro de ese núcleo pedagógico con una "articulación comprensiva entre experiencia y conocimiento" (Coiduras, Paris y Torrelles, 2014, p. 31). Esta articulación tiene relación directa con el concepto de competencia que incide en el saber hacer. Elmore (2010) subraya que en términos de evaluación es preciso considerar la funcionalidad del aprendizaje fuera del aula donde se conjuga el binomio enseñanza-aprendizaje, con el fin de evaluar el rendimiento de los estudiantes y la calidad educativa a través de la funcionalidad de ese aprendizaje en el mundo laboral.

$\mathrm{El} \mathrm{foco} \mathrm{a} \mathrm{donde} \mathrm{debe} \mathrm{dirigirse} \mathrm{la} \mathrm{evaluación} \mathrm{es} \mathrm{el} \mathrm{docente} \mathrm{porque} \mathrm{ejerce} \mathrm{de} \mathrm{puente} \mathrm{entre}$ estudiante y contenido como "agente modulador" (Bolívar y Bolívar, 2011, p. 7). Además, la evaluación debe "reflejar los atributos personales que se requiere utilizar para interactuar eficientemente y tener éxito en el desempeño de una tarea en un contexto social determinado" (García, Loredo, Luna y Rueda, 2008, p. 99). Esto implica la necesidad de introducir el término desempeño docente porque determina las dimensiones que se deben evaluar. Este término, al igual que el de competencia, ha sido trasladado desde el entorno empresarial al ámbito educativo (Cerda, 2000) y, por tanto, parece coherente plantear la evaluación del desempeño en términos de competencias alcanzadas por cada estudiante. Montenegro (2003, p. 18) define el desempeño docente "como el cumplimiento de sus funciones; éste se haya determinado por factores asociados al propio docente, al estudiante y al entorno". Por consiguiente, conviene que en una adecuada evaluación del desempeño docente se tengan en cuenta tanto los elementos personales involucrados, como aquellos referidos al contexto que pueden influir en los resultados. 
La concepción educativa basada en competencias (Le Boterf, 2000), determina que la evaluación puede plantearse de acuerdo a dos paradigmas, según los resultados (paradigma positivista) y según los aspectos cualitativos (paradigma interpretativo); ambos están relacionados con las dos funciones de la evaluación mencionadas, formativa y sumativa. En palabras de Ardoino (2005, p. 96), la diferencia entre ellos no radica únicamente en "una epistemología de la prueba se trata de una epistemología del testimonio". Sería razonable pensar entonces que pueden ser complementarios.

Un enfoque positivista acepta la suficiencia de una evaluación del rendimiento del estudiante como indicio de calidad susceptible de ser utilizado como producto, dado que se basa casi únicamente en la medición de resultados (Carbajosa, 2011; Delgado, 1995). Algunos informes de evaluación de los estudiantes pueden resultar herramientas útiles en este sentido (PISA, TIMSS, PIRLS), dado que responden a "procedimientos rigurosos en su diseño y construcción” (González, Caso, Díaz y López, 2012, p. 53). El informe PISA (2009, 2012), por ejemplo, permite realizar un ranking para medir los resultados de aprendizaje de los estudiantes, aunque su objetivo prioritario es comparar sistemas educativos, no la evaluación del desempeño de los estudiantes. Conviene señalar que este tipo de evaluación estandarizada únicamente "tendrá efectos positivos si es concebida, percibida y empleada como un mecanismo de responsabilidad pública de todos los actores vinculados al quehacer educativo" (Ravela et al., 2008, p. 48), aspecto que hace que el docente deba también estar de acuerdo con su planteamiento, algo que no siempre sucede. No obstante, este planteamiento cuantitativo, recogido en forma de resultado, se plantea como una evaluación terminal, centrada en los resultados del alumno, soslayando las demás personas implicadas y otros elementos a tener en cuenta. Este carácter ulterior dado a la evaluación impide uno de sus principios básicos, la retroalimentación (Fuentes y Herrero, 1999; Pérez-Gómez, 2009; Ryan, Scott, Freeman y Patel, 2002). Pensando en la evaluación en estos términos, son fundamentales las acciones que se desarrollan en los centros educativos que pueden facilitar retroalimentación, tales como elemento de satisfacción personal, clima institucional propicio o mejoras académicas y administrativas (Rueda, 2008).

Los diferentes sectores de la comunidad educativa parecen coincidir en la necesidad de una evaluación mediante la incorporación de suficientes fuentes de información (Fernández, 2010; Marcelo, 2009) que garanticen la disposición de datos tanto de carácter cuantitativo como cualitativo. Sin embargo, encontramos visos de controversia en la forma de hacer efectiva esa recogida de resultados y aún más en las consecuencias que estos pueden tener, convirtiendo la evaluación "en uno de los objetos de mayor tensión y disputa entre los actores del escenario educativo de cada país" (Román y Murillo, 2008, p. 3), dado que en algunos casos la evaluación se percibe como una herramienta para fiscalizar y realizar comparaciones (Álvarez, 2008). Sin duda, este modo de proceder conlleva la desconfianza del profesorado ante cualquier control (Benedito, 1992). Puede ser pertinente que la política educativa plantee la evaluación de forma procesual, como un medio de fomentar la cooperación y no la competitividad entre los diferentes agentes e instituciones educativas (Sahlberg, 2009). Por tanto, es precisa una concienciación del profesorado de la importancia de una evaluación planteada como cultura, como punto de mejora y en aras de la calidad académica.

Una vez situado el docente en el centro de la evaluación, hemos de definir qué elementos son susceptibles de ser evaluados y sobre todo para qué. Según Zabalza (1990), son dos 
enfoques, el perfeccionamiento y el control, que pueden resultar antagónicos; sin embargo, es difícil delimitar los elementos a evaluar en el docente dentro de estas dos utilizaciones de los resultados a obtener, dada la interacción entre todos los elementos y la complejidad de la tarea que realiza.

\subsection{El foco en la evaluación del docente}

El foco de la evaluación del desempeño docente debe tener distintos alcances, complementarios entre sí y que den como resultado un indicador de la calidad global de la tarea realizada. El primer alcance se centraría en las relaciones del docente en la institución, "construir un ambiente propicio en pos de un proyecto" (Montenegro, 2003, p. 15), que tiene un claro carácter colectivo, por lo que el sentido desde la recogida de resultados incluiría un modelo de evaluación que va a determinar los niveles del clima en el centro.

La recogida de datos debe plantearse desde el equipo directivo, dado que los resultados van a definir los niveles de clima en el centro, siendo esta una de las responsabilidades de aquellos que dirigen la institución, evitando unir la evaluación del profesorado con la organización de la escuela, porque son realidades diferentes (Natrielo, 1990). El docente debe conjugar lo que el currículo oficial exige y la realidad del contexto donde ejerce su labor; estos datos también deben ser incluidos en la evaluación. Los equipos directivos deben facilitar, como complemento a estos datos, tiempos y espacios que faciliten la autoevaluación del docente (Amores, Moral y Ritacco, 2015).

Los objetivos planteados en la evaluación del docente pueden ser muchos y variados y tienen además que utilizarse como elementos no disjuntos; deberían encaminarse al crecimiento del docente, conocer si ha llegado a las competencias mínimas exigibles y planificar un sistema de promoción. El equilibrio está en lograr que la evaluación no sea amenazante para el docente y que tanto el objetivo de su aplicación como las consecuencias de sus resultados formen parte de un proceso de transparencia, que tenga como fin la mejora de calidad de alguno de los elementos o situaciones del escenario educativo. Bacharach (1989, citado en Valdés, 2000) nombra cuatro principios para la evaluación por desempeño que podrían determinarnos los tipos de evaluación, ya que al analizarlos pueden darnos las claves para conocer distintas maneras de abordar la forma de llevar a cabo este proceso y la explotación de los resultados.

El primero de los principios prioriza una evaluación basada en capacidades frente al rendimiento, dirigida al profesor directamente y no a resultados del proceso; de esta manera pueden prevenirse situaciones como el teaching to the test, elemento que ha dirigido la enseñanza de algunos profesores al entrenamiento de sus estudiantes para la capacitación en pruebas de evaluación estandarizadas (Bertola y Checchi, 2012; Moos, 2013). El segundo, desarrollo frente a uniformidad, proyecta una evaluación particular para cada docente o grupo de docentes. El tercero, subjetivo frente a objetivo, no plantea una situación dicotómica, sino una necesidad de conjugar ambos aspectos de forma que se complementen aportando información que garantice la utilidad de la evaluación con unos resultados que sirvan como antesala de acciones de mejora. En este punto, se encuentran los modelos de valor añadido, teacher value added models, considerando una medida que valore la calidad en términos de mejora entre el antes y el después del periodo de enseñanza. Por último, formativa versus sumativa esboza una evaluación de recorrido frente a una holística final. 
El modelo propuesto lo hemos recogido en la Figura 1, donde se muestran aquellos puntos que consideramos necesario integrar en el planteamiento de esta evaluación del docente, teniendo en cuenta los dos sentidos de la evaluación mencionados (positivista e interpretativo).

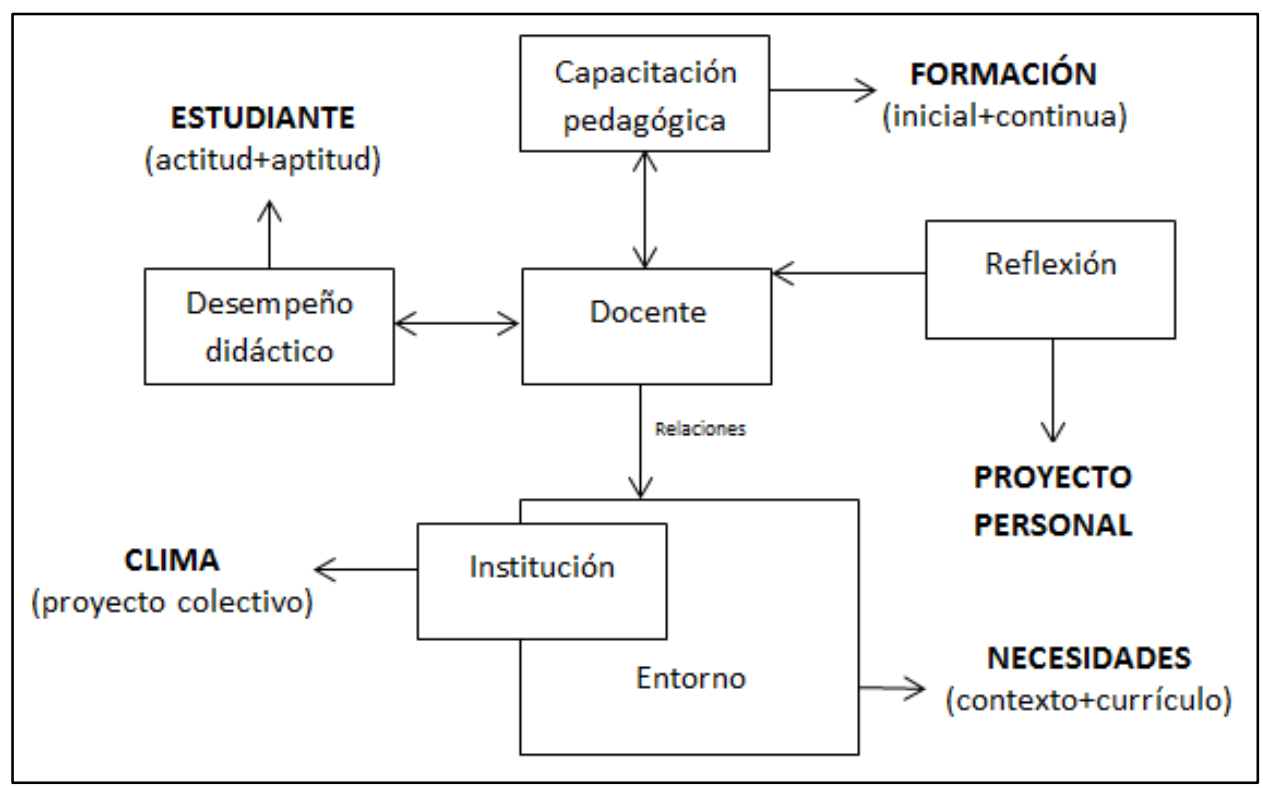

Figura 1. Elementos en la evaluación del docente Fuente: Elaboración propia.

En la evaluación del docente es preciso separar la capacitación pedagógica del desempeño didáctico. La evaluación de la capacitación pedagógica debería plantearse desde dos perspectivas, una inicial y otra continua o procesual. La inicial debería tener en cuenta la titulación recibida y la naturaleza específica de las materias a impartir; la procesual formaría parte del reciclaje necesario en la formación y especialización de los docentes en ejercicio. Como resultado de esta evaluación, podrían plantearse planes formativos desde los centros o las administraciones de carácter personalizador, que garanticen por parte del docente un dominio del conocimiento pedagógico del contenido, sustentado siempre en las particularidades de su disciplina.

El desempeño didáctico se basa en la capacidad del docente para planificar, desarrollar y evaluar el proceso de aprendizaje del estudiante como receptor, cuyos resultados deberían centrarse tanto en actitud como en aptitud, teniendo en cuenta que "la aptitud para aprender supone combinar los conocimientos previos sobre la materia y el interés, persistencia y compromiso con el aprendizaje” (Arteaga, 2006, p. 16). La evaluación del desempeño didáctico debe basarse en resultados objetivos, tanto provenientes de la evaluación de rendimiento de los estudiantes, como de elementos procesuales y contextuales que permitan la retroalimentación y la toma de decisiones durante todas las fases del proceso de aprendizaje.

Esta evaluación del desempeño no puede olvidar que el docente es una persona con sus aspiraciones, motivaciones, autoestima o satisfacción (Fernández, 1995). Estos factores entran en juego en su desempeño diario, sirviendo también como elemento de mejora cuando los niveles son óptimos. Sin embargo, no es algo que se enseñe en las escuelas de 
formación del profesorado, quizá porque se olvida la relación que tiene con las estrategias de aprendizaje. Esta evaluación debe ponerse en práctica como un proceso de auto-reflexión, "el análisis de las tareas del profesor en el aula como el estudio de sus vivencias de la enseñanza, dan a conocer que se preocupa y desarrolla una intensa labor" (Rosales, 2000, p. 14). El resultado de este proceso integra la construcción del proyecto personal del docente como parte de la madurez personal y profesional.

Por tanto, dentro de esta evaluación específica de las capacidades didácticas y pedagógicas, sería de interés medir la destreza del docente para motivar al estudiante a aprender, lo que determinará una mejora y unos buenos resultados finales en términos de rendimiento y de actitud. Consideramos que esta ha de ser la auténtica clave de la mejora profesional. No obstante, hay una dificultad añadida porque no está claro cuáles pueden ser los indicadores que permitan establecer una medición de esta destreza en el docente.

$\mathrm{El}$ aspecto formativo responde a una necesidad de definir marcos para un buen ejercicio de la profesión que faciliten al docente herramientas que le permitan adaptarse a las necesidades de cada situación particular de aprendizaje. Es importante señalar que una formación para el docente, como resultado de un proceso evaluador, para que sea efectiva debe formar parte de un proceso de autorreflexión enmarcado dentro de su proyecto personal. El docente debe participar en el diseño de esta evaluación para garantizar la efectividad de estos planes formativos, la evaluación pactada logra una mayor implicación del docente, dado que no se percibe como una amenaza (Iwanicki, 1997).

Otro de los efectos de los resultados de la evaluación es la mejora de ascenso o retributiva, asociada en gran parte de los países únicamente a la antigüedad, lo que puede suponer que docentes experimentados dejen el aula ocupando puestos superiores en la escala jerárquica (Mizala y Romaguera, 2003). Además, estos sistemas pueden emplear estándares competitivos que en algunos casos lleven al rechazo por parte de los docentes a la hora de la cooperación y el trabajo conjunto en los claustros, "los estándares competitivos son claramente inapropiados en un sistema de evaluación diseñado para facilitar el crecimiento profesional” (Bacharach, Conely y Shedd, 1997, p. 194). No obstante, este sistema es válido para los profesionales más destacados, pero quizá no lo sea para el resto, que se verían desmotivados ante la imposibilidad de obtener una compensación económica por el desempeño profesional.

Analizando las prioridades asignadas a uno u otro tipo de evaluación consideramos que ha de encontrarse un modelo que conjugue, en la medida de lo posible, aspectos que puedan adecuarse a la evaluación contextualizada en cada realidad. En función de la utilización de los datos procedentes de la evaluación del docente, los principales modelos de evaluación docente se basan en dos tipos de uso, formación y retribución.

\section{Asturias, comunidad pionera en la evaluación del docente en España}

Algunos países han regulado la evaluación de los docentes en los niveles educativos obligatorios con parámetros que los docentes conocen previamente. Un ejemplo es el 
trabajo de Murillo (2007), donde se analiza en qué países de Europa y América se ha regulado esta evaluación del profesorado no universitario, de manera formal (Figura 2).

\begin{tabular}{|c|c|c|c|c|c|}
\hline \multicolumn{6}{|l|}{ AMÉRICA } \\
\hline Argentina & Si & El Salvador & No & Paraguay & No \\
\hline Bolivia & Si & EEUU, California & Sí & Perú & Sí \\
\hline Brasil & No & EEUU, Carolina del N. & Sí & Puerto Rico & Sí \\
\hline Chile & Sí & Guatemala & Sí & Rep. Dominicana & Sí \\
\hline Colombia & Si & Honduras & Si & Uruguay & Sí \\
\hline Costa Rica & Sí & México & Sí & Venezuela & Sí \\
\hline Cuba & Sí & Nicaragua & No & & \\
\hline Ecuador & No & Panamá & No & & \\
\hline \multicolumn{6}{|l|}{ EUROPA } \\
\hline Alemania & Si & Finlandia & No & Malta & Si \\
\hline Austria & No & Francia & No & Noruega & No \\
\hline Bélgica, Francófona & No & Grecia & Sí & Países Bajos & No \\
\hline Bélgica, Flamenca & No & Hungría & No & Polonia & Sí \\
\hline Bélgica, Valona & No & Irlanda & No & Portugal & Sí \\
\hline Chipre & No & Islandia & No & Reino U.* & Sí \\
\hline Dinamarca & No & Italia & No & Reino U., Escocia & No \\
\hline Eslovaquia & No & Letonia (Latvia) & No & República Checa & Sí \\
\hline Eslovenia & Si & Liechstenstein & No & Rumania & Sí \\
\hline Estonia & Si & Lituania & No & Suecia & No \\
\hline España & No & Luxemburgo & No & & \\
\hline
\end{tabular}

Figura 2. Países con sistemas generalizados de evaluación del desempeño docente de carácter externo

Fuente: Murillo (2007, p. 90).

Lo más significativo de la tabla 1 son los países que "sî" tienen regulada la evaluación planteada en términos de mejora del desempeño docente $y$, por tanto, de la calidad del sistema, teniendo como efecto primordial "una perfección progresiva de la actividad profesional del docente” (Schulmeyer, 2002, p. 37). Este modo de proceder está encaminado a facilitar el desarrollo profesional. España aparece como uno de los países que carece de esta evaluación.

La primera ley en España que recoge explícitamente la evaluación del docente es la Ley Orgánica de Educación, LOE (2006), que vincula la evaluación con la rendición de cuentas, procedimiento integral "para exigir responsabilidad por sus resultados a los actores del sistema" (Gajardo, 2012, p. 11). Por otra parte, la actual Ley 8/2013, para la Mejora de la Calidad Educativa, LOMCE, otorga en su artículo 132 la responsabilidad de la evaluación del docente al director del centro, en términos de colaboración en la realización de la misma. No obstante, no hace mención a la forma u obligación de la acción evaluadora sobre el profesorado, algo que puede dar libertad a las distintas administraciones educativas de las comunidades autónomas a organizar esta acción, definiendo pautas e indicadores adaptados a su realidad más cercana.

En el contexto de España, el Principado de Asturias ha sido pionero en la regulación de la evaluación de la función docente. El camino se inicia con la firma de un acuerdo en 2006 entre la Administración del Principado de Asturias y las organizaciones sindicales 
representadas en la Mesa General de Negociación. El marco del acuerdo se centró en el desarrollo de la carrera profesional del personal estatutario del Servicio de Salud, pero incluye el compromiso de la Administración para "negociar modelos de desarrollo profesional para el resto del personal” (Viceconsejería de Presupuestos y Administración Pública, 2006, p. 2).

Este compromiso estaba pendiente de la aprobación definitiva del Estatuto básico del Funcionario docente no universitario, en un proceso de negociación del Ministerio de Educación con el resto de la comunidad educativa. El borrador de la ley incluía el artículo 30, centrado específicamente en la carrera profesional de los docentes. Sin embargo, la firma de este estatuto no se llegó a ratificar; en cambio, sí que se aprueba en abril del 2007 la Ley 7/2007, del Estatuto Básico del Empleado Público (EBEP). Dicho texto normativo, en su artículo 2 , establece que el personal docente:

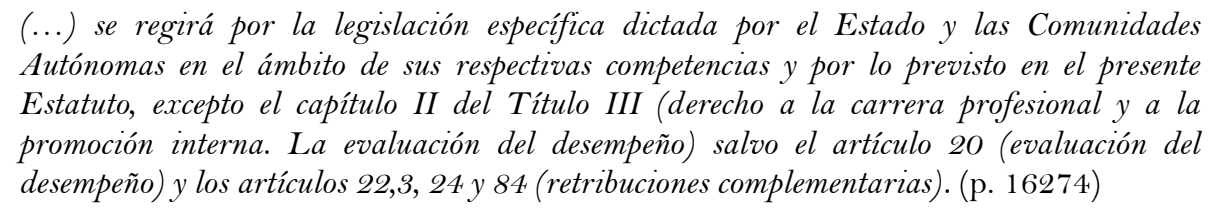

Por lo tanto, el EBEP no regulaba la carrera profesional de los docentes y emplazaba al desarrollo específico de su normativa a través de la competencia delegada a las Comunidades Autónomas.

Ante esta falta de desarrollo de un marco normativo estatal, la Administración del Principado de Asturias decidió impulsar el reconocimiento de la función docente. Además, el interés radicaba en que esta medida supondría una clara mejora de las condiciones laborales y del reconocimiento profesional de este personal. No obstante, la única vía normativa real para la aprobación y puesta en práctica del proceso de evaluación de la función docente fue la de fundamentarse en lo previsto en la Ley Orgánica de Educación 2/2006, LOE, y también sobre la base del primigenio Acuerdo sobre carrera y desarrollo profesional de los empleados públicos del Principado de Asturias de diciembre de 2006. En atención a esto se inició un proceso de negociación con los sindicatos docentes para articular un marco normativo autonómico que permitiera implantar un sistema de evaluación que sería complementado y sustituido, en su caso, por la norma estatal básica cuando esta se aprobara (es decir, por el Estatuto Básico del Docente o similar).

Desde el principio el desarrollo de este sistema de evaluación docente ha sido apoyado por parte de las organizaciones sindicales con representación en la Mesa Sectorial de Personal Docente, en concreto, por FETE UGT y ANPE (organizaciones que ostentan la mayoría en dicha Mesa Sectorial). Estas plasmaron su apoyo en el "Acuerdo por el que se establecen medidas tendentes a la implantación del sistema de evaluación de la función docente y sus incentivos", de 5 de noviembre de 2009, a partir del cual la Administración educativa presentó para su aprobación en la Junta General del Principado de Asturias la Ley del Principado de Asturias 6/2009, de 29 de diciembre, de Evaluación de la Función Docente y sus Incentivos.

La Dirección General de Personal de la Consejería de Educación definió los indicadores, partiendo de: 
$\checkmark$ El análisis de las tareas docentes fuera del aula y relacionadas con el funcionamiento general del centro.

$\checkmark$ El desarrollo de las relaciones con la comunidad educativa.

$\checkmark$ La coordinación e implicación con los diferentes órganos de gobierno y coordinación de los centros.

$\checkmark$ Los aspectos más evidentes relacionados con la implicación, responsabilidad y cumplimiento de cualquier trabajador.

La evaluación es planteada mediante diferentes fichas de evaluación adaptadas, en la medida de lo posible, a las principales categorías o ámbitos en los que trabaja el personal docente. En el trabajo interno se elaboró una lista amplia de ítems a incluir en estas fichas de la que se seleccionaron, para este primer plan, los más claros y elementales. Hay que tener en cuenta el carácter provisional de esta normativa. Por eso, quedaba pendiente su desarrollo en un segundo plan dirigido a matizar, modificar y ampliar algunos ítems respecto a los ya incluidos en el primero, utilizando para ello el análisis estadístico de las pruebas piloto realizadas.

El instrumento de evaluación de los docentes de este modelo se estructura de acuerdo a tres apartados, para cada uno de los cuales se recoge una valoración global. Estos apartados corresponden a tres dimensiones del desempeño: formación, mayor dedicación al centro y participación en la consecución de objetivos conseguidos en la Programación General Anual (PGA). De acuerdo a los elementos que se proponían para evaluar en el docente, en la figura 1, este diseño de evaluación da cabida fundamentalmente al proyecto colectivo de la institución y a la capacitación pedagógica del docente en términos de actualización formativa. La evaluación de cada profesor, solicitada de forma voluntaria, es realizada por el director del centro.

Los ítems de este instrumento responden al modelo de Perrenoud (2004), que establece diez competencias, de las cuales se han seleccionado ocho. No se evalúan aquellas competencias que estarían más relacionadas con la situación de aprendizaje y el trabajo de los estudiantes, apoyándose en investigaciones previas que aconsejan no basarse únicamente en indicadores de resultados (Jornet, González-Such y Bakieva, 2012). La distribución de ítems por bloque y competencia se recoge en la tabla 1 y el instrumento original se incluye en el anexo 1, donde se podrá conocer la descripción completa de la etiqueta.

La respuesta a todos los bloques se realiza de manera dicotómica, logrado versus no logrado, obteniendo una valoración global por cada uno de los bloques. Respecto al bloque correspondiente a la dedicación al centro, se considerará superado si se obtiene una valoración positiva en al menos el 50\% de los ítems. También lo es si nueve de los ítems de la dedicación al centro (II) han alcanzado una valoración positiva. La valoración global es positiva si se alcanza en dos de los tres bloques. El resultado inmediato de una evaluación total positiva es la obtención de un incentivo económico, que dependerá del subgrupo laboral al que pertenece, y siendo únicamente prescriptivo hasta la siguiente evaluación. 
Tabla 1. Distribución de ítems por competencias en el instrumento de evaluación del desempeño docente.

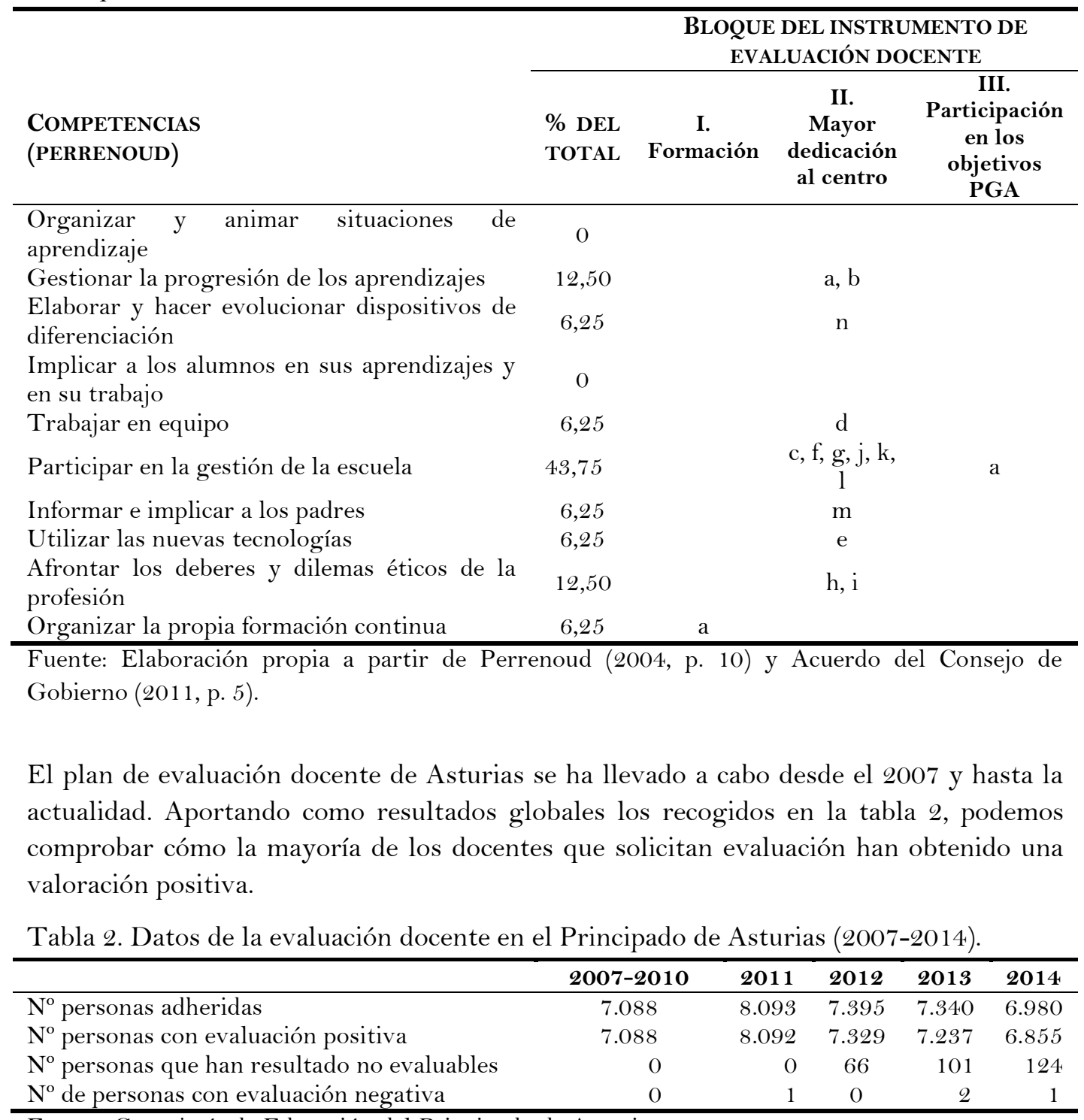

Fuente: Consejería de Educación del Principado de Asturias.

El Principado de Asturias cuenta actualmente con 408 centros docentes. El número total de funcionarios y funcionarias de carrera es de 7670, lo que implica que el $91 \%$ del funcionariado de carrera está adherido al plan de evaluación de la función docente. Para aquellas personas que no superan esta evaluación voluntaria, se realiza un plan personalizado para dar respuesta a las necesidades de mejora que cada uno de ellos manifiesta.

Este proceso de evaluación responde a un modelo positivista, con resultados cuantificados al término del proceso, incidiendo como hitos de importancia en la formación las necesidades del contexto y el proyecto colectivo del Centro, de acuerdo con Willet (1994, citado en Navarro, 2014, p. 6) cuando defiende la idea de que "midiendo el cambio individual es posible informar sobre el progreso de cada persona y, 
consecuentemente, evaluar la efectividad de los sistemas educativos". En este sentido, Bacharach, Conely y Shedd (1997) apuntan que:

(...) la búsqueda de criterios de evaluación siempre más objetivos -y de estándares para establecer distinciones objetivas entre los distintos niveles de mérito respecto de un criterio dado- es una absoluta necesidad a la hora de conceder subidas salariales de una forma justa y equilibrada. (p. 188)

Sin embargo, cabe mencionar lo exiguo de este processo, no pudiendo consolidar sus resultados por la ausencia de una legislación estatal que facilite la promoción en un cuerpo de funcionarios, el docente, sin estructura que regule la carrera profesional.

\section{La necesidad de un estatuto docente en España}

El presente apartado se traza como una síntesis, subrayando que, como medida para la mejora de la calidad del sistema educativo, convenga dotar de un marco regulador la evaluación docente en España. En este punto, el artículo 103.3 de la Constitución Española establece que las condiciones de trabajo de los funcionarios públicos deberán articularse por medio de un estatuto propio con rango de ley. En el caso de los docentes, este estatuto debería regular los derechos y las obligaciones. No obstante, la aprobación de un estatuto implicaría la profesionalización de la docencia, lo que supondría introducir un cierto carácter competitivo entre los docentes. Esto únicamente sería justificable si conllevase una clara mejora de la calidad educativa.

Respecto a los antecedentes, en 2007 hubo un intento de aprobar un estatuto del funcionario docente no universitario a propuesta del Partido Popular, que gobernaba. El borrador, inclúa la evaluación positiva de la actividad docente para la promoción interna, constituyendo así la carrera docente. En 2011, los dos partidos mayoritarios Partido Socialista y Partido Popular- habían incluido en su programa electoral la realización de una prueba para el acceso a la carrera docente, un MIR educativo. Al respecto, López-Rupérez (2014) señala que la calidad de un sistema educativo está en relación con el procedimiento establecido de acceso a la misma. Según un estudio desarrollado por el Consejo General de los Ilustres Colegios Oficiales de Doctores y Licenciados en Filosofía y Letras y en Ciencias, el 15,6 \% de los profesores encuestados está totalmente de acuerdo con la implantación de esta prueba de selección, el 29,5\% bastante de acuerdo y el $21 \%$ algo de acuerdo (Valle y Manso, 2014). Estos datos confirman que el acuerdo favorable a la implantación de este sistema no es muy amplio.

En este sentido, Darling-Hammond (2001) subraya que para los políticos, preocupados por mejorar la enseñanza, existen dos posibilidades: burocratizarla o profesionalizarla. El quid de la cuestión es la elección entre un control externo (burocratización) o interno, de cada profesor (profesionalización). Sin embargo, esta alternativa es relevante porque en función de cuál sea la elección afectaría al tipo de personas que se sentirían atraídas por la enseñanza. Fernández-Enguita (2001) explica las características de estos modelos (Tabla 3) para la organización docente a los que denomina: burocrático y liberal.

Sin embargo, Fernández-Enguita (2001) no ratifica ninguno de estos sistemas y plantea mitigar el carácter autoritario del Estado con un modelo igualitario y participativo. Aboga por un estatuto profesional para la docencia no impuesto desde arriba, sino aceptado y anclado con el compromiso de los profesores. Esto subraya lo que advierte 
Bolívar (2008), que para la eficacia de la evaluación docente no conviene un control externo, burocrático, es preferible otro más centrado en el sujeto.

Tabla 3. Modelos para la organización de la profesión docente.

\begin{tabular}{llll}
\hline \multicolumn{1}{c}{ MODELO BUROCRÁtICO } & \multicolumn{3}{c}{ MODELO LIBERAL } \\
\hline Disciplina & $\begin{array}{l}\text { Autonomía } \\
\text { Disponibilidad al servicio de la institución } \\
\text { Jurisdicción } \\
\text { competencial } \\
\text { Integración en el cuerpo }\end{array}$ & $\begin{array}{l}\text { Ejercicio liberal de la profesión } \\
\text { "Lo que diga el público, pero solo si puede pagarlo” }\end{array}$ \\
\hline "Todo para el público, pero sin el público” & de & desarrollo \\
\hline Fuente: Fernández-Enguita (2001). & &
\end{tabular}

La burocratización de la docencia acarrea consecuencias, según Darling-Hammond (2001); entre ellas la principal es que se soslayaría a las personas, tanto a los educadores como a los educandos. Asimismo, es uno de los síntomas de insatisfacción entre los docentes y un motivo para abandonar la profesión (Darling-Hammond, 2001; Esteve, 2006). Igualmente, podría conllevar el peligro de uniformidad de la actividad docente, con el añadido riesgo de reducir la enseñanza a una transmisión de información, olvidando el aspecto formativo de la educación.

En este punto, Finlandia, uno de los primeros países según el ranking del informe PISA, ha optado por incrementar la profesionalización como antídoto contra la burocratización (Bolívar, 2012). Según Enkvist (2010), han tratado de impedir que el juicio de los administradores reemplace al de los docentes para evitar que las personas con talento para la enseñanza ignoren su vocación docente. En este sentido, Darling-Hammond (2001) señala que las investigaciones realizadas respecto a la influencia de las políticas en los docentes son útiles para advertir cuál es la relación de los profesores con los estudiantes, aspecto necesario para conocerlos mejor de cara a motivarlos para el aprendizaje.

Igualmente, el estatuto docente debe constituir un reconocimiento de los buenos docentes para lograr que los mejores profesionales estén interesados en dedicarse a enseñar. La docencia es un arte que trata con personas cuyo objetivo es sacar de ellas lo mejor, pero esto únicamente se logrará si se atrae a la docencia a los mejores. Por tanto, parece conveniente que el modelo de organización docente debe atender a lo que interesa a los buenos profesores. Además, la aprobación del estatuto docente debe implicar la sistematización de la evaluación del profesorado (Sclan y Darling-Hammond, 1992).

En definitiva, el estatuto docente ha de fomentar la responsabilidad del docente, no debe limitarse a una clasificación, sin promover la motivación para evitar la habituación del profesorado. Sin duda, la aprobación del estatuto docente ha de contemplar la planificación de una adecuada formación del profesorado, desarrollada en su mayoría en los propios centros siguiendo las propuestas de las teorías adaptativas, de forma "que les implique competentemente en el diseño de soluciones y recursos" (Arteaga y García, 2008, p. 256). Esto conlleva necesariamente que debe estar abierta a la aportación del docente, que es quien mejor conoce cuáles son sus intereses y sus deficiencias. 


\section{Conclusiones}

A modo de conclusión, en este trabajo se pone el énfasis en dos cuestiones prioritarias: la necesidad de una evaluación del desempeño docente y la regulación normativa del acceso a la profesión. La cuestión no es si conviene o no la evaluación docente, aspecto que ha sido aceptado, sino cuáles son los elementos a tener en cuenta para realizarla. La evaluación en función de los resultados obtenidos por los estudiantes, volcada en lo cuantitativo, parece insuficiente porque sería una evaluación final, olvidando la relevancia de la evaluación formativa. Se ha realizado una propuesta de los elementos que constituyen el foco del desempeño docente, que han de tenerse presentes para una adecuada evaluación. Estos elementos dan un carácter holístico a la tarea desempeñada, desde las relaciones del docente con la institución a la que pertenece, su capacitación y desempeño didáctico como acciones complementarias que repercuten directamente en los resultados de los estudiantes, y su propio proyecto personal, todos ellos planteados de una manera alineada.

Además, la eficacia de la evaluación conlleva su aceptación por el docente y que esté encaminada a acrecentar la motivación de aquel en el ejercicio profesional.

En España, Asturias ha sido la comunidad pionera en diseñar un plan para la evaluación docente, basado en ocho competencias, estableciendo una relación de los logros obtenidos en cada una de ellas con incentivos económicos para el profesorado. Este modelo valora la formación, la dedicación al centro, la participación y la capacitación pedagógica de los docentes, pero conviene que todo proceso de evaluación culmine en un plan de mejora personalizado de cada docente. Planteamos conveniente como medida de futuro para nuestra investigación una validación del cuestionario en el que se fundamenta el modelo atendiendo a sus propiedades psicométricas.

Respecto a la regulación de la profesión, se analizan dos modelos: la burocratización o la profesionalización. El exceso de burocracia es rechazado por los docentes y, además, soslaya a las personas; por eso, el segundo es más apropiado para atraer a los mejores profesionales a la docencia. En este sentido, se ha propuesto, siguiendo la iniciativa de Asturias, la aprobación de un estatuto docente, que debería ser elaborado con la participación de los propios docentes. Sin duda, esta medida establecerá un modelo de evaluación del desempeño docente con el fin de descubrir aspectos a mejorar que deben ser contemplados por los planes de formación del profesorado.

Se plantea con visión prospectiva ofrecer un diagnóstico de la percepción que tienen los docentes en niveles previos a la universidad sobre el modelo propuesto, atendiendo a las distintas etapas educativas y contextos de los centros.

\section{Referencias}

Acuerdo del Consejo de Gobierno. (2011). Aprobación del primer plan de evaluación de la función docente. Boletín Oficial del Principado de Asturias, 62, 1-12.

Álvarez, J. M. (2008). Evaluar el aprendizaje de una enseñanza centrada en competencias. En J. Gimeno (Comp.), A. I. Pérez, J. B. Martínez, J. Torres, F. Angulo y J. M. Álvarez, Educar por competencias, ¿̇qué hay de nuevo? (pp. 207-233). Madrid: Morata. 
Amores, J., Moral, C. y Ritacco, M. (2015). El desarrollo de procesos de autoevaluación como capacidad del liderazgo pedagógico. Un estudio en Educación Secundaria en Andalucía. Revista Iberoamericana de Evaluación Educativa, 8(2), 57-76.

Anderson, J. A. (2005). Accountability in education. París: UNESCO.

Ardoino, J. (2005). Complejidad y formación. Pensar la educación desde una mirada epistemológica. Buenos Aires: Novedades Educativas.

Arteaga, B. (2006). La educación adaptativa: Una propuesta para la mejora del rendimiento en matemáticas de los alumnos de enseñanza secundaria obligatoria. (Tesis Doctoral, Universidad Complutense de Madrid, Madrid).

Arteaga, B. y García, M. (2008). La formación de competencias docentes para incorporar estrategias adaptativas en el aula. Revista Complutense de Educación, 19(2), 253-274.

Bacharach, A. B., Conely, S. C. y Shedd, J. B. (1997). Cómo evaluar al profesorado para promociones y aumentos salariales. En J. Millman y L. Darling-Hammond (Dirs.), Manual para la evaluación del profesorado (pp. 188-207). Madrid: La Muralla.

Benedito, V. (Coord.). (1992). La formación del profesorado universitario. Madrid: Subdirección general de formación del profesorado, MEC.

Bertola, G. y Checchi, D. (2012). Motivación, organización y carrera de los docentes: El caso italiano. Revista de Política Educativa, 3, 35-62.

Bolívar, A. (2008). Evaluación de la práctica docente. Una revisión desde España. Revista Iberoamericana de Evaluación Educativa, 1(2), 56-74.

Bolívar, A. (2012). El proceso de burocratización de la escuela: Una rigidez que impide el incremento de profesionalidad y no se corresponde con la enseñanza y el aprendizaje hoy. Crítica, 982, 28-32.

Bolívar, A. y Bolívar, M. (2011). La didáctica en el núcleo del mejoramiento de los aprendizajes. Entre la agenda clásica y actual de la Didáctica. Perspectiva Educacional, 50(2), 3-25.

Carbajosa, D. (2011). Debate desde paradigmas en la evaluación educativa. Perfiles Educativos, 33(132), 181-190.

Cerda, H. (2000). La evaluación como experiencia total. Bogotá: Magisterio.

Coiduras, J., Paris, G. y Torrelles, C. (2014). La evaluación de competencias en una experiencia de formación dual de maestros: Diferencias y semejanzas entre tutores de escuela y de universidad. Estudios Pedagógicos, 40, 29-48. doi:10.4067/s0718-07052014000200003

Coll, C., Colomina, R., Onrubia, J. y Rochera, M. J. (1992). Actividad conjunta y habla: Una aproximación al estudio de los mecanismos de influencia educativa. Infancia y Aprendizaje, 15(59-60), 189-232. doi:10.1080/02 103702.1992.10822356

Darling-Hammond, L. (2001). El derecho de aprender. Buenas escuelas para todos. Barcelona: Ariel.

De la Orden, A. (2012). Innovación, evaluación y calidad en la educación. REVALUE. Revista de Evaluación Educativa, 1(1), 1-21.

De la Vega, L. F. (2015). Accountabilty y mejoramiento educativo: Análisis de experiencias internacionales. Educação y Realidade, 4O(1), 275-298. doi:10.1590/2075-623645060

Delgado, K. (1995). Evaluación y calidad de la educación. Nuevos aportes, procesos y resultados. Lima: Cooperativa Editorial Magisterio.

Elmore, R. (2010). Mejorando la escuela desde la sala de clases. Santiago de Chile: CAP. 
Enkvist, I. (2010). La educación en peligro. Pamplona: Eunsa.

Esteve, J. M. (2006). La profesión docente en Europa: Perfil, tendencias y problemática. La formación inicial. [Comentarios a los Informes EURYDICE y OCDE sobre la cuestión docente]. Revista de Educación, 340, 19-40.

Fernández, F. J. (2010). Sentido y necesidad de la evaluación de la práctica docente. Avances en Supervisión Educativa: Revista de la Asociación de Inspectores de Educación de España, 13, 5.

Fernández, M. (1995). Los ciclos vitales de los profesores. Granada: Force.

Fernández-Enguita, M. (2001). A la busca de un modelo profesional para la docencia: ¿Liberal, burocrático o democrático? Revista Iberoamericana de Educación, 25, 43-64.

Fuentes, M. E. y Herrero, J. R. (1999). Evaluación docente: Hacia una fundamentación de la autoevaluación. Revista Electrónica Interuniversitaria de Formación del Profesorado, 2(1), 353368.

Gajardo, M. (2012). La educación tras dos décadas de cambio. ¿Qué hemos aprendido?¿¿ué debemos transformar? Santiago de Chile: Programa de Promoción de la Reforma Educativa en América Latina y el Caribe (PREAL).

García, B., Loredo, J., Luna, E. y Rueda, M. (2008). Modelo de la evaluación de competencias docentes para la educación media y superior. RIEE. Revista Iberoamericana de Evaluación Educativa, 1(3), 97-108.

Gimeno, J. y Pérez, A. (1989). La enseñanza: Su teoría y su práctica. Madrid: Akal.

Gimeno, J. (2010). La carrera por el personal profesional. Interuniversitario. Journal of Teacher Education, 68, 243-260.

González, C., Caso, J., Díaz, K. y López, M. (2012). Rendimiento académico y factores asociados: Aportaciones de algunas evaluaciones a gran escala. Bordón. Revista de pedagogía, 64(2), 51 68.

Houssaye, J. (1993). Le triangle pédagogique, ou comment comprendre la situation pédagogique. En J. Houssaye (Ed.), La Pédagogie, une encyclopédie pour aujourd'hui (pp. 13-24). París: ESF Editeur.

Imbernón, F. (1994). La formación y el desarrollo profesional del profesorado: Hacia una nueva cultura profesional. Barcelona: Graó.

Iwanicki, E. (1997). Evaluación del profesorado para la mejora de escuelas. En J. Millman y L. Darling-Hammond (Dir.), Manual para la evaluación del profesorado (pp. 222- 239). Madrid: La Muralla.

Jornet, J. M., González-Such. J. y Bakieva, M. (2012). Los resultados de aprendizaje como indicador para la evaluación de la calidad de la docencia universitaria. Reflexiones metodológicas. RIEE. Revista Iberoamericana de Evaluación Educativa, 5(2), 99-115.

Le Boterf, G. (2000). Ingeniería de las competencias. Barcelona: Gestión 2000.

España. (2007). Ley 7/2007, de 12 de abril, del Estatuto Básico del Empleado Público.

España. (2006). Ley Orgánica 2/2006, de Educación.

España. (2009). Ley 6/2009, de 29 de diciembre, de Evaluación de la Función Docente y sus Incentivos.

España. (2013). Ley Orgánica 8/2013, de 9 de diciembre, para la mejora de la calidad educativa.

López-Rupérez, F. (2014). Fortalecer la profesión docente: Un desafío crucial. Madrid: Narcea. 
Marcelo, C. (2009). La evaluación del desarrollo profesional docente. En C. V. Vélaz Medrano y D. Vaillant (Eds.), Aprendizaje y desarrollo profesional docente (pp. 119-128). Madrid: Organización de Estados Iberoamericanos para la Educación, la Ciencia y la Cultura.

Mizala, A. y Romaguera, P. (2003). El sistema nacional de evaluación del desempeño docente (SNED) en Chile. Washington D. C.: Banco Interamericano de Desarrollo.

Montenegro, I. A. (2003). Evaluación del desempeño docente. Fundamentos, modelos e instrumentos. Bogotá: Magisterio.

Moos, L. (2013). School leadership in a contradictory world. Revista de Investigación Educativa, 31(1), 15-29. doi:10.6018/rie.31.1.162511

Murillo, F. J. (2007). Evaluación del desempeño y carrera profesional docente. Santiago de Chile: OREALC/UNESCO.

Natrielo, G. (1990). Intended and unintended consequences: Purposes and effects of teacher evaluation. En J. Millman y L. Darling-Hammond (Eds.), The New Handbook of Teacher Evaluation: Assessing elementary and secondary school teachers (pp. 35-45). Newbury Park: Corwin Press.

Navarro, E. (2014). El valor añadido en educación: Cuestiones teóricas y metodológicas. (Tesis doctoral, Universidad Complutense de Madrid, Madrid).

OCDE. (2009). Evaluación y reconocimiento de la calidad de los docentes. Prácticas Internacionales. París: Autor.

Pérez-Gómez, Á. I. (2009). La evaluación como aprendizaje. Córdoba: Ediciones AKAL.

Perrenoud, P. (2004). Diez nuevas competencias para enseñar. Invitación al viaje. Barcelona: Graó.

PISA. (2009). Programa para la Evaluación Internacional de los Alumnos. París: OCDE.

PISA. (2012). Programa para la Evaluación Internacional de los Alumnos. París: OCDE.

Ravela, P., Arregui, P., Valverde, G., Wolfe, R., Ferrer, G., Martínez Rizo, F., Aylwin, M. y Wolff, L. (2008). Las evaluaciones educativas que América Latina necesita. Revista Iberoamericana de Evaluación Educativa, 1(1), 45-63.

Román, M. y Murillo, F. J. (2008). La evaluación del desempeño docente: Objeto de disputa y fuente de oportunidades en el campo educativo. Revista Iberoamericana de Evaluación Educativa, 1(2), 1-6.

Rosales, C. (2000). Evaluar es reflexionar sobre la enseñanza. Madrid: Narcea.

Rueda, M. (2008). La evaluación del desempeño docente en las universidades públicas en México. RIEE. Revista Iberoamericana de Evaluación Educativa, 1(3), 8-17.

Rueda, M. (2009). La evaluación del desempeño docente: Consideraciones desde el enfoque por competencias. Revista Electrónica de Investigación Educativa, 11(2), 1-16.

Ryan, S., Scott, B., Freeman, H. y Patel, D. (2000). The Virtual University. Londres: Kogan Page.

Sahlberg, P. (2009). Learning first: School accountability for a sustainable society. En K. D. Gariepy, B. L. Spencer y J. C. Couture (Eds.), Educational Accountability. Profesional voices from the field (pp. 1-22). Rotterdam: Sense Publishers.

Schulmeyer, A. (julio, 2002). Estado actual de la evaluación docente en trece países de América Latina. Ponencia presentada en la Conferencia Regional El desempeño de los maestros en América Latina y el Caribe: Nuevas prioridades, Brasilia. 
Sclan, E. M. y Darling-Hammond, L. (1992). Beginning teacher performance evaluation: An overview of state policies. Trends and Issues. Washington: ERIC Clearinghouse on Teacher Education. American Association of Colleges for Teacher Education.

Scriven, M. (1967). The methodology of evaluation. En R. W. Tyler, R. M. Gagné y M. Scriven (Eds.), Perspectives of curriculum evaluation (pp. 39-83). Chicago, IL: Rand McNally and Company.

Stiggins, R. J. y Duke, D. (1988). The case for commitment to teacher growth research on teacher evaluation. Nueva York, NY: State University of New York Press.

Valdés, H. (mayo, 2000). Evaluación del desempeño docente. Ponencia presentada en el Encuentro Iberoamericano sobre Evaluación del Desempeño Docente, México.

Valle, J. M. y Manso, J. (2014). La voz del profesorado: Acceso a la profesión docente e inserción en el puesto de trabajo. Madrid: CGCDL.

Viceconsejería de Presupuestos y Administración Pública. (2006). Acuerdo sobre carrera y desarrollo profesional de los empleados públicos del principado de Asturias. Oviedo: Dirección General de la Función Pública.

Zabalza, M. A. (1990). Evaluación orientada al perfeccionamiento. Revista Española de Pedagogía, 48(186), 295-317. 


\section{Anexo 1. Modelo de ficha de evaluación de la función docente}

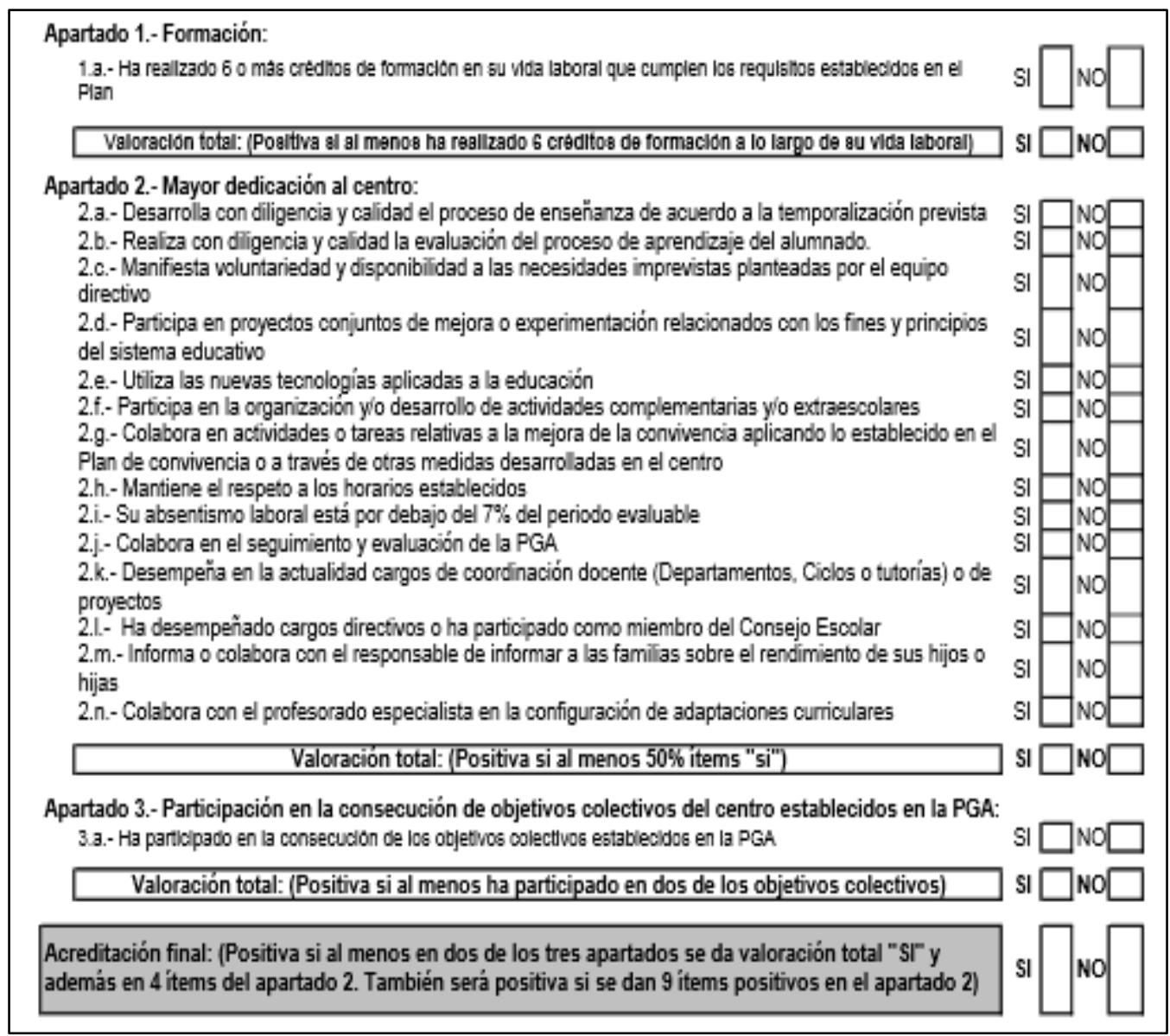

Fuente: Acuerdo del Consejo de Gobierno (2011, p. 5). 


\section{Breve CV de los autores}

\section{Blanca Arteaga Martínez}

Licenciada en CC. Matemáticas por la Universidad Autónoma de Madrid y Doctora en CC. de la Educación por la Universidad Complutense de Madrid. Dedicada a la docencia en distintos niveles educativos previos a la universidad, ha combinado esta labor con otros espacios y niveles educativos, siendo profesora asociada en la Universidad Carlos III de Madrid y la Saint Louis University in Madrid. En la actualidad, imparte docencia en distintos grados y máster, en la Universidad Internacional de la Rioja (UNIR), donde dirige un grupo de investigación, Educación Personalizada en la Era Digital. Su investigación se ha centrado en la investigación-acción en el aula, tomando la didáctica de la matemática como eje vertebrador. Ha dirigido y participado en distintos proyectos de investigación subvencionados y proyectos de innovación educativa con distintas universidades. Integrante del grupo de investigación Pedagogía Adaptativa de la Universidad Complutense de Madrid. E-mail: blanca.arteaga@unir.net

\section{Josu Ahedo Ruíz}

Licenciado en Filosofía, Ciencias Políticas, Derecho y graduado en Comunicación. Doctor en Filosofía por la Universidad de Navarra. Docente en colegios concertados de enseñanza secundaria durante dieciséis años. Coordinador académico del área de trabajo social de la Universidad Internacional de la Rioja (2011-2013). Desde 2012 es Vicerrector de Estudiantes y de Calidad Académica de la UNIR. Ha impartido docencia en asignaturas en el área de la metodología de investigación, antropología y orientación familiar. Ha publicado varios artículos en metodología de investigación y en el ámbito de la filosofía de la educación. Actualmente ha centrado su investigación en la educación del carácter y de la voluntad. 